\title{
Influence of Adopted Nutation Model on VLBI NEOS-Intensives Analysis
}

\author{
Oleg Titov \\ Saint-Petersburg University, Astronomical Institute, Bibliotechnaya sq., \\ 2, Petrodvorets, Saint-Petersburg, Russia, 198904
}

\section{Introduction}

Very Long Baseline Interferometry (VLBI) provides information about the Earth's rotation. Various observational programs have operated for different specific purposes since 1983. The purpose of the NEOS-Intensive program is to make observations of Universal Time (UT1-UTC). Short observational sessions (1-2 hours) include only the transcontinental baseline (Wettzell - NRAO20). 20-30 individual scans are performed during the observational time. Only five parameters, wet delay for the reference station, wet delay for the second station, clock offset for the second station, clock rate for the second station, and UT1-UTC are considered in the parametric model.

The purpose of this paper is to investigate the influence of the adopted nutation model on the UT1-UTC estimates. VLBI data from the NEOS-Intensives have been analyzed using the OCCAM 3.4 software. All reduction calculations are in accordance with the IERS Conventions 1996 (McCarthy, 1996).

Conventional weighted least-squares method (LSM) is used for the VLBI data analysis. Let's consider the following parametric model

$$
A x+w=h,
$$

where $A$ is the matrix of partial derivatives, $h$ the vector of observations, $w$ the vector of observational errors, and $x$ the vector of estimated parameters. The vector $x$ can be estimated as follows.

$$
\hat{x}=\left(A^{T} Q_{w}^{-1} A\right)^{-1} A^{T} Q_{w}^{-1} h,
$$

where $Q_{w}^{-1}$ is the inverted covariance matrix of observational errors.

\section{Discussion}

The IERS Conventions (McCarthy, 1996) recommends two nutation models for implementation ("old" IAU 1980 model and a "new" one, provided by Thomas Herring). Fig. 1 shows corrections to the IAU 1980 nutation model which have been taken from the IERS $97 \mathrm{C} 04$ time series. Fig. 2 demonstrates corrections to the Herring's model obtained from analysis of NEOS-A VLBI data within 1999. It seems the latter corrections are essentially smaller than the former ones.

The radiosource coordinates are not absolutely correct. As a result the shortcomings of the a priori IAU 1980 nutation model bias estimates of other 

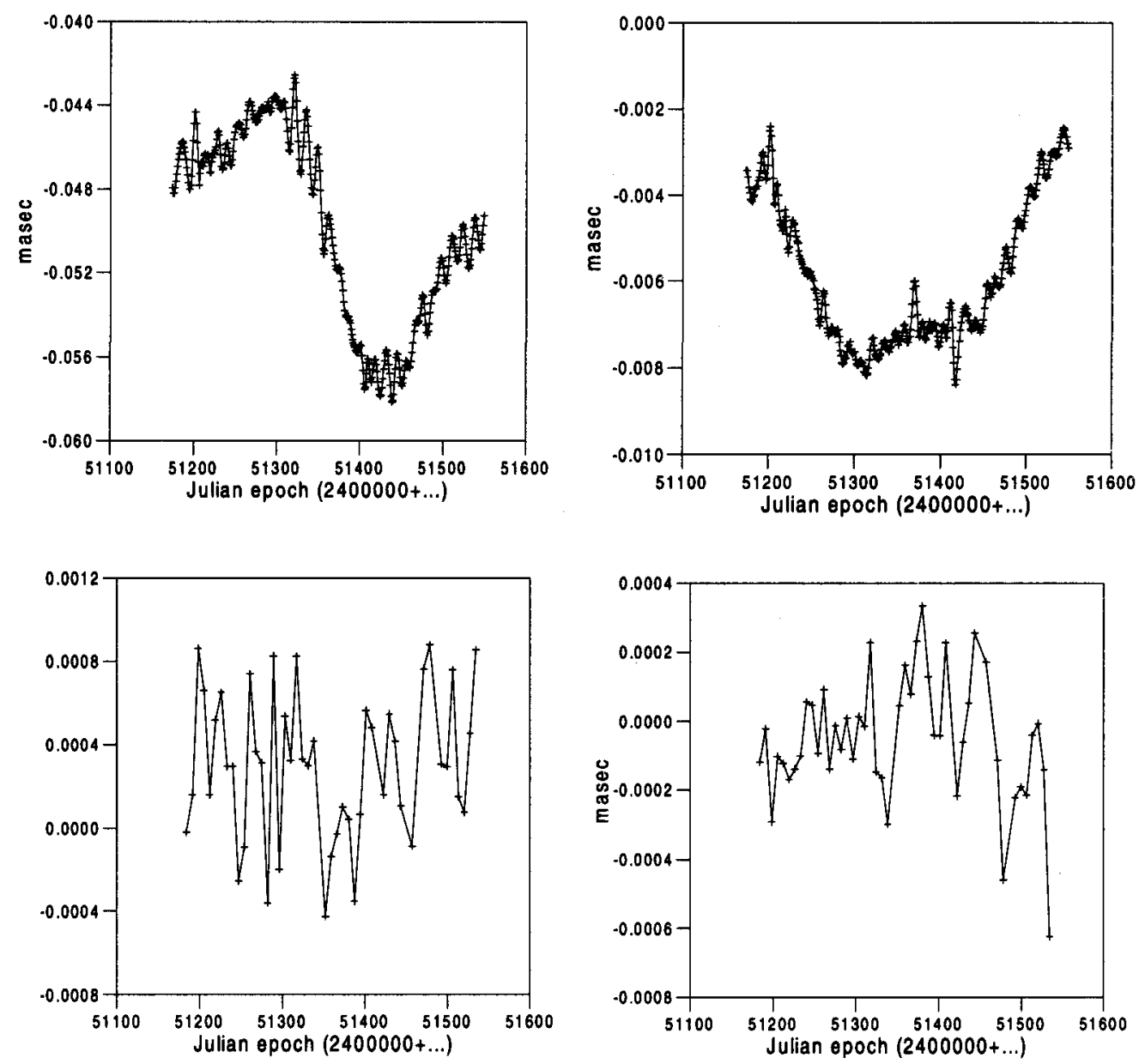

Figure 1. Corrections to IAU 1980 nutation model in $\Delta \psi$ (top, left) and $\Delta \epsilon$ (top, right) and to Herring's nutation model (bottom, left) and $\Delta \epsilon$ (bottom, right). 


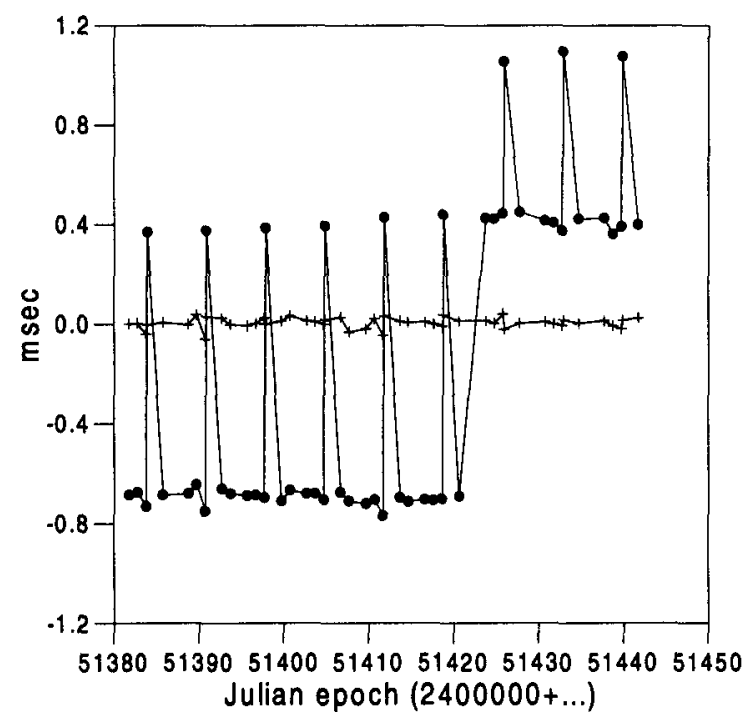

Figure 2. Variations of UT1-UTC from NEOS-Intensives using IAU 1980 nutation model (black dots) and Herring's nutation model (crosses).

parameters under adjustment. The bias can be seen as a break in the UT1-UTC time series every 3 months due to regular changes in list of the radiosources. Additionally, $\mathrm{XT}$ sessions provide a regular break with respect to the XU sessions every week for the same reason. Fig. 2 demonstrates the structure of the breaks in the UT1-UTC time series within a 60-day period from 22-Jul-1999 till 23Sep-1999. The biased estimates are shown by black dots. Hefty \& Gontier (1997) published the analogous picture using the IAU 1980 nutation model for reductions. Herring's nutation model provides more correct results. For comparison the model has been applied in the adjustment of the same observational NEOSIntensives data. The resulting time series of the UT1-UTC values is shown hy crosses in Fig. 2, and, in another scale, in Fig. 3. Therefore implementation of more adequate nutation model reduces the bias of the UT1-UTC estimates.

The Herring nutation model demonstrates a perfect accuracy. Nevertheless, the corrections obtained from analysis of NEOS-A sessions contain obvious systematic effects. It means that any advanced model for nutation will provide in the future even more excellent estimates of UT1-UTC from VLBI data.

Overall, we have to use very accurate reductions to avoid possible bias of UT1-UTC estimates due to the incompleteness of the parametric model (1). The radiosource coordinate's uncertainty will not be a dramatic problem if an adequate nutation model is applied for reductions. 


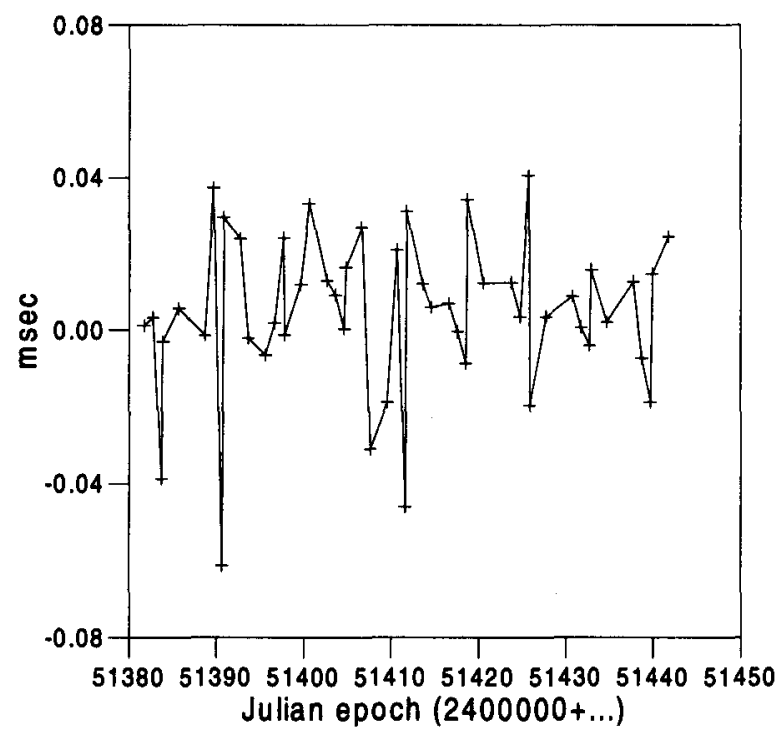

Figure 3. Variations of UT1-UTC from NEOS-Intensives using Herring's nutation model.

\section{References}

Hefty J., Gontier, A.-M., 1997, Sensitivity of UT1 determined by single-baseline VLBI to atmospheric delay model, terrestrial and celestial reference frame, Journal of Geodesy, 71, 253-261.

McCarthy D., 1996, IERS Conventions, Paris Observatory. 